\title{
Pump-Power-Driven Mode Switching in a Microcavity Device and Its Relation to Bose-Einstein Condensation
}

\author{
H. A. M. Leymann, ${ }^{1,2,{ }^{*}}$ D. Vorberg, ${ }^{2, \dagger}$ T. Lettau, ${ }^{1}$ C. Hopfmann, ${ }^{3}$ C. Schneider, ${ }^{4}$ M. Kamp, ${ }^{4}$ \\ S. Höfling, ${ }^{4,5}$ R. Ketzmerick, ${ }^{2,6}$ J. Wiersig, ${ }^{1}$ S. Reitzenstein, ${ }^{3}$ and A. Eckardt ${ }^{2, \$}$ \\ ${ }^{1}$ Institut für Theoretische Physik, Otto-von-Guericke-Universität Magdeburg, \\ Postfach 4120, D-39016 Magdeburg, Germany \\ ${ }^{2}$ Max-Planck-Institut für Physik komplexer Systeme, Nöthnitzer Strasse 38, D-01187 Dresden, Germany \\ ${ }^{3}$ Institut für Festkörperphysik, Technische Universität Berlin, \\ Hardenbergstraße 36, D-10623 Berlin, Germany \\ ${ }^{4}$ Technische Physik and Wilhelm Conrad Röntgen Research Center for Complex Material Systems, \\ Physikalisches Institut, Universität Würzburg, Am Hubland, D-97074 Würzburg, Germany \\ ${ }^{5}$ SUPA, School of Physics and Astronomy, University of St Andrews, \\ St Andrews KY16 9SS, United Kingdom \\ ${ }^{6}$ Technische Universität Dresden, Institut für Theoretische Physik and Center for Dynamics, \\ D-01062 Dresden, Germany
}

(Received 22 December 2016; revised manuscript received 7 April 2017; published 22 June 2017)

We investigate the switching of the coherent emission mode of a bimodal microcavity device, occurring when the pump power is varied. We compare experimental data to theoretical results and identify the underlying mechanism based on the competition between the effective gain, on the one hand, and the intermode kinetics, on the other. When the pumping is ramped up, above a threshold, the mode with the largest effective gain starts to emit coherent light, corresponding to lasing. In contrast, in the limit of strong pumping, it is the intermode kinetics that determines which mode acquires a large occupation and shows coherent emission. We point out that this latter mechanism is akin to the equilibrium Bose-Einstein condensation of massive bosons. Thus, the mode switching in our microcavity device can be viewed as a minimal instance of Bose-Einstein condensation of photons. Moreover, we show that the switching from one cavity mode to the other always occurs via an intermediate phase where both modes are emitting coherent light and that it is associated with both superthermal intensity fluctuations and strong anticorrelations between both modes.

DOI: 10.1103/PhysRevX.7.021045

\section{INTRODUCTION}

The development of optical cavities [1-4] has led to (laser) devices with an almost vanishing lasing threshold $[5,6]$. Bimodal (micro)lasers have been realized in various types of systems, such as ring lasers [7,8], vertical-cavity surface-emitting lasers [9], a quantum-dot micropillar variant [10,11], and 2D photonic crystal cavity lasers [12]. In these systems, the switching of the mode showing coherent emission [9,13-18] has gathered substantial interest because of potential technical applications, such as optical flip-flop memories, tunable sensitive

\footnotetext{
"ham.leymann@gmail.com

†v@pks.mpg.de

teckardt@pks.mpg.de
}

Published by the American Physical Society under the terms of the Creative Commons Attribution 4.0 International license. Further distribution of this work must maintain attribution to the author(s) and the published article's title, journal citation, and DOI.
Subject Areas: Photonics, Quantum Physics, Statistical Physics switches $[19,20]$, and a simple realization of nonequilibrium phase transitions [21,22].

In this article, we study the switching of the coherent emission mode in bimodal micropillar lasers occurring when the pump power is ramped up. By comparing experimental data to theoretical results based on a phenomenological model, we find that the basic mechanism underlying the mode switching is the competition between effective gain, on the one hand, and the intermode kinetics, on the other. Namely, the mechanism that selects which of the modes shows coherent emission is found to be fundamentally different for weak pumping (just above the threshold) and in the limit of strong pumping. For weak pumping, the selected mode (i.e., the mode selected for coherent emission) is characterized by the largest effective gain, and coherent emission corresponds to lasing. In contrast, for strong pumping, the selected mode depends neither on the coupling to the gain medium nor on the loss rates of both modes. Instead, it is determined completely by the intermode kinetics, i.e., by processes that transfer photons from one mode to the other. We show that this 


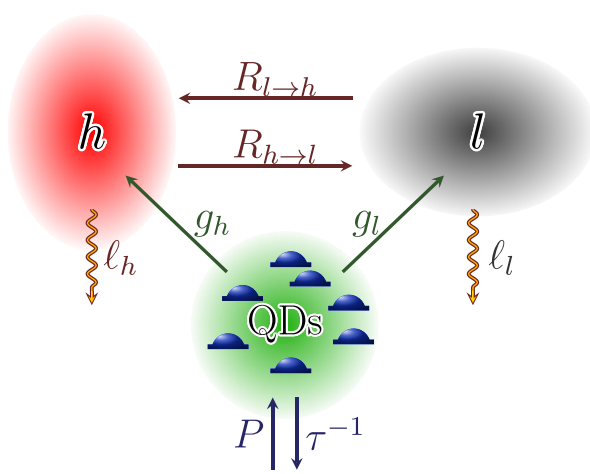

FIG. 1. Illustration of the relevant processes in our bimodal system. The intermode kinetics between the modes $h$ and $l$ is determined by the rates $R_{i \rightarrow j}$. The modes lose their photons with rates $\ell_{i}$. This loss is compensated by new excitation from the quantum dots (QDs) with gain rates $g_{i}$. The quantum dots are pumped with rate $P$, and they lose their excitations spontaneously with rate $\tau^{-1}$.

mechanism is formally identical to the one leading to Bose-Einstein condensation of an ideal gas of massive bosons (i.e., with a conserved particle number) in contact with a thermal bath. Therefore, the mode switching in our system can be viewed as a minimal instance of Bose-Einstein condensation of photons.

The question of whether a system of photons (or bosonic quasiparticles) with nonconserved particle number can undergo Bose-Einstein condensation in a similar way as a thermal gas of massive bosons has raised considerable interest in the last decade. Here, the problem to be overcome is to achieve a quasiequilibrium situation, where a single mode acquires a macroscopic occupation via a thermalizing kinetics, despite the nonequilibrium nature of the system resulting from particle loss to be balanced by pumping. Beautiful experiments, showing that such a situation can indeed be achieved, have been conducted in systems of exciton-polaritons [23-29], magnons [30-33], and photons in dye-filled cavities [34]. While the microcavity device investigated in this article is simpler than these systems, in the sense that it is described in terms of two relevant modes only, it captures one of the most important aspects of photon condensation in a minimal fashion, namely, that the condensate mode is selected not by pumping but rather by the kinetics of the photons.

The starting point for our theoretical description of a bimodal micropillar is a generic phenomenological master equation describing the relevant processes of the system $[10,35]$ : the coupling between the pumped medium and the cavity modes, loss, and the intermode kinetics (Fig. 1). Such birth-death models have also been used to study (analogs of Bose-Einstein condensation in) population dynamics [36,37], transport [38,39], and networks [40], as well as quantum gases of massive bosons $[41,42]$. This approach, which is different from the microscopic modeling pursued in former studies of
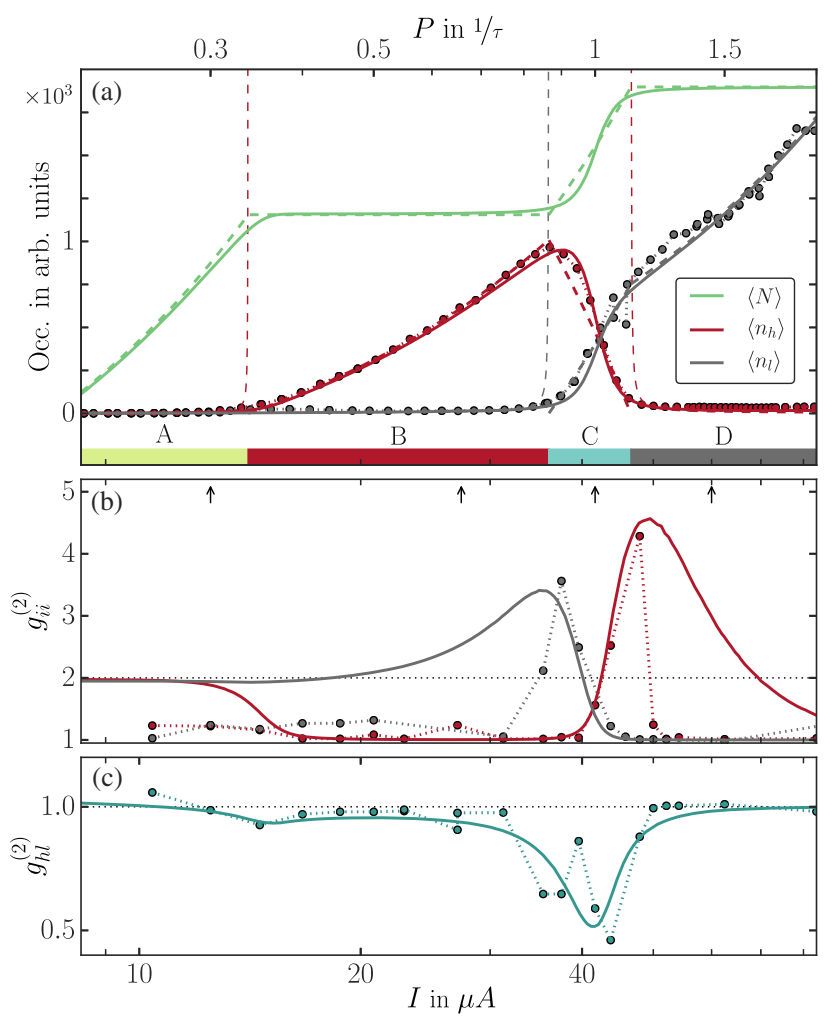

FIG. 2. Measured and calculated microcavity input-output characteristics (high-effective-gain mode in red, low-effectivegain mode in gray). The injection current $I$ is proportional to the pump rate $P$. Here, circles (connected to guide the eye), solid curves, and dashed curves refer to experimentally measured data, exact Monte Carlo results, and asymptotic mean-field theory, respectively. Panel (a) depicts the mean occupations of the modes and the number of excited carriers (light green) in arbitrary units. The colored bars at the bottom of this panel mark the phases determined by the asymptotic theory (see Sec. IV C). Panel (b) shows the autocorrelation $g_{i i}^{(2)}$ for both modes in comparison with the value 2 expected for thermal emission (dotted black line). Panel (c) depicts the cross-correlation $g_{h l}^{(2)}$ in comparison to the value 1 for correlated emission (dotted black line). For the theoretical curves, we use $s=0, G=0.77$, as well as (in units of the spontaneous loss rate $\left.\tau^{-1}\right) g_{h}=1.6 \times 10^{-3}, g_{l}=2.1 \times 10^{-3}$, $\ell_{h}=2.2 \times 10^{-2}, \quad \ell_{l}=3.8 \times 10^{-2}, \quad R_{l \rightarrow h}=1.7 \times 10^{-4}, \quad$ and $A_{h \rightarrow l}=8.5 \times 10^{-6}$ (see Appendix A).

bimodal (micro) lasers $[8,10,19,43-47]$ and interacting exciton-polariton systems (e.g., Refs. [48-50]), provides excellent agreement with the experimental data (Fig. 2). In order to treat these equations analytically, we work out an approximation scheme that combines the theory of Bose selection, which was recently developed to describe (nonequilibrium) Bose condensation of ideal gases of massive bosons with conserved particle number [41], with particle loss and the coupling to a pumped reservoir (gain medium). We justify this approximation by exact numerical simulations. Somewhat counterintuitively, our theory shows that it is the limit of strong pumping, where 


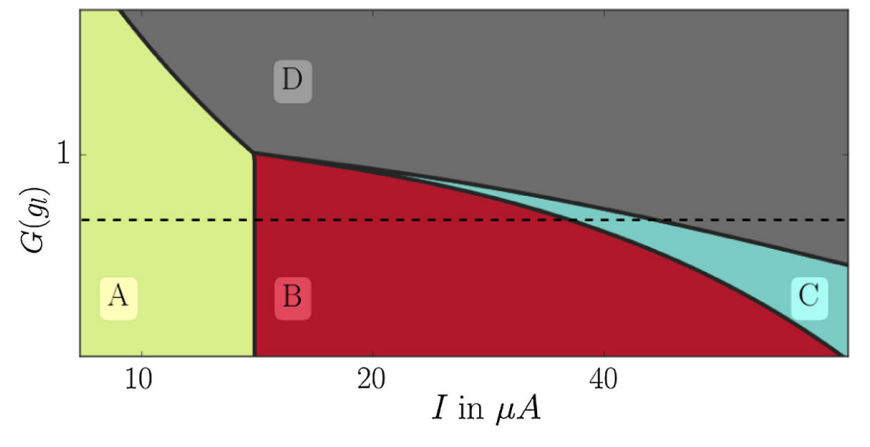

FIG. 3. Phase diagram with phase A for no selected mode, phase $\mathrm{B}$ when mode $h$ is selected, phase $\mathrm{C}$ when both modes are selected, and phase D when mode $l$ is selected. Plotted versus the injection current $I$ that is proportional to pump rate $P$ and the ratio of the effective-gain rates $G=g_{l} \ell_{h} / g_{h} \ell_{l}$ with fixed $g_{h} \ell_{l} / \ell_{h}$. Except for $g_{l}$, all parameters are the same as in Fig. 2. The dashed line indicates the value of $g_{l}$ used in Fig. 2. While for $G<1$ all phases are present when increasing the pump rate $P$, for $G>1$ the mode $l$, which is favored by the direct mode coupling, actually becomes the high-effective-gain mode; thus, no mode switching occurs.

the selected mode is determined by the intermode kinetics, that is described by terms that are formally identical to those appearing in the description of massive bosons and their equilibrium condensation. Furthermore, we find that the mode switching occurs via an intermediate phase where both modes are coherently emitting (see phase diagram in Fig. 3).

We also investigate the statistical properties of our system. In bimodal lasers, where one mode dominates the emission for all pump rates, the nonlasing mode usually exhibits superthermal intensity fluctuations $[7,10]$, and the emission of both modes is strongly anticorrelated $[8,46,51-53]$. In the situation where mode switching occurs, we find that the superthermal intensity fluctuations of the nonselected mode and strong anticorrelations occur whenever a mode starts or ceases to be selected. We show that these experimentally observed statistical properties can be described theoretically by an effective reduction of the spontaneous intermode transitions caused by mode interactions.

This paper is organized as follows. In Sec. II, the experimental setup is presented. The theoretical description in terms of a master equation is introduced in Sec. III. An analytical theory of the mode switching and its relation to Bose-Einstein condensation is then worked out in Sec. IV. Finally, in Sec. V, we investigate the statistical properties of the system, before coming to the conclusions in Sec. VI.

\section{EXPERIMENT}

Electrically pumped quantum-dot micropillars are fabricated by the etching of a planar AlAs/GaAs-distributed Bragg reflector $\lambda$ cavity in which a single active layer of self-assembled $\mathrm{In}_{0.3} \mathrm{Ga}_{0.7}$ As quantum dots is embedded centrally [54]. A detailed description can be found in Ref. [55]. The micropillar used in this study has a diameter of $3.0 \mu \mathrm{m}$. Because of the strong confinement of light, the micropillars exhibit a spectrum of discrete modes. The fundamental mode $H E_{1,1}$ is composed of two orthogonally linearly polarized components, which are ideally energetically degenerate. In reality, however, asymmetries in the manufacturing process, which results in slightly elliptical structures, lift the energetical degeneracy of the fundamental modes $[1,56]$. The resulting mode splitting of the micropillar used in this study is $(42 \pm 2) \mu \mathrm{eV}$. Besides a finite mode splitting, the two fundamental modes also exhibit slightly different quality factors, $14000 \pm 1500$ and $12500 \pm 1500$, respectively. The different spectral and local overlaps of the modes with the gain medium and the mode polarization alter their coupling to the quantumdot emitters. As a consequence, the former mode (mode $h$ ) is characterized by a higher effective gain [i.e., gain-loss ratio, see Eq. (4)] than the latter one (mode $l$ ).

A high-resolution $(25 \mu \mathrm{eV})$ micro-electroluminescence setup is used to characterize the micropillars spectrally at cryogenic temperatures of $15 \mathrm{~K}$. A linear polarizer, as well as a $\lambda / 4$ plate in front of the monochromator, enables polarization-resolved spectroscopy. For statistical analysis via the autocorrelation function $g_{i i}^{(2)}(\tau=0)$ with zero delay time for the emission, a fiber-coupled Hanbury-Brown and Twiss (HBT) setup with a temporal resolution of $40 \mathrm{ps}$ is used. For measuring the equal-time cross-correlation function $g_{h l}^{(2)}(\tau=0)$ between the orthogonally polarized micropillar modes, the emission is selected by a polarization maintaining a 50/50 beam splitter, and the split beam is directed to a second identical spectrometer-with the polarizer in front of the second monochromator oriented orthogonally to the first. These equal-time correlations are defined by

$$
g_{i j}^{(2)}(\tau=0)=\frac{\left\langle\hat{b}_{i}^{\dagger} \hat{b}_{j}^{\dagger} \hat{b}_{i} \hat{b}_{j}\right\rangle}{\left\langle\hat{b}_{i}^{\dagger} \hat{b}_{i}\right\rangle\left\langle\hat{b}_{j}^{\dagger} \hat{b}_{j}\right\rangle} \equiv g_{i j}^{(2)},
$$

where $\hat{b}_{i}$ is the bosonic annihilator operator of mode $i$. The emission characteristics of the CW-pumped micropillar are shown in Fig. 2. In panel (a), the output characteristics of modes $h$ (red) and $l$ (gray) are shown as a function of the injection current $I$ (quantifying the pumping strength). Up to about $13 \mu \mathrm{A}$ (phase A), both modes are below threshold and show a small increase in output emission only. Between $13 \mu \mathrm{A}$ and $36 \mu \mathrm{A}$ (phase $\mathrm{B}$ ), the mode $h$ is selected, and its emission increases strongly while that of mode $l$ remains small. Between $36 \mu \mathrm{A}$ and $50 \mu \mathrm{A}$ (phase $\mathrm{C}$ ), both modes are selected, but while the emission intensity of mode $l$ increases, that of mode $h$ decreases. Beyond $50 \mu \mathrm{A}$ (phase $\mathrm{D}$ ), only mode $l$ is selected. In Figs. 2(b) and 2(c), the zero-delay autocorrelation $g_{i i}^{(2)}$ 
and cross-correlation $g_{h l}^{(2)}$ functions are plotted. Note that, because of finite temporal resolution of the HBT setup and the low coherence time of the modes, we could not properly resolve $g_{i i}^{(2)}$ experimentally for values above 1 in phases A and $\mathrm{B}$ [57].

\section{MASTER EQUATION}

Our starting point for the theoretical description of the system is a phenomenological master equation for the probabilities $\rho_{N}^{n}$ to find the system in a state with $N$ excited emitters and photon numbers $\boldsymbol{n}=\left(n_{h}, n_{l}\right)$ in the high- and the low-effective-gain mode. It takes the form

$$
\frac{d}{d t} \rho_{N}^{n}=C_{\text {laser }}(\rho)+C_{\text {kin }}(\rho)
$$

and shall be solved for the steady state obeying

$$
\frac{d}{d t} \rho_{N}^{n}=0
$$

The first term on the right-hand side of the master equation (2) describes how photons leave and enter the cavity modes via loss and coupling to the emitters:

$$
\begin{aligned}
C_{\text {laser }}(\rho)= & P\left[\rho_{N-1}^{n}-\rho_{N}^{n}\right]-\tau^{-1}\left[N \rho_{N}^{n}-(N+1) \rho_{N+1}^{n}\right] \\
& -\sum_{i} g_{i}\left[N\left(n_{i}+1\right) \rho_{N}^{n}-(N+1) n_{i} \rho_{N+1}^{n-\mathbf{e}_{i}}\right] \\
& -\sum_{i} l_{i}\left[n_{i} \rho_{N}^{n}-\left(n_{i}+1\right) \rho_{N}^{n+\mathbf{e}_{\mathbf{i}}}\right]
\end{aligned}
$$

where $P$ and $\tau^{-1}$ denote the pump and the loss rate of the emitters, respectively, $g_{i}$ quantifies the gain of cavity mode $i$ from the emitters, and $\ell_{i}$ is the loss rate of cavity mode $i$. An additional or removed photon in mode $i$ is denoted by $\pm \mathbf{e}_{\mathbf{i}}$, [i.e., $\boldsymbol{e}_{h}=(1,0), \boldsymbol{e}_{l}=(0,1)$ ]. The modes $h$ and $l$ are defined by the higher and lower effective gain, $g_{i} / l_{i}$, respectively, so that we obtain an effective-gain ratio

$$
G \equiv \frac{g_{l} / \ell_{l}}{g_{h} / \ell_{h}}<1 .
$$

The terms contained in $C_{\text {laser }}(\rho)$ are sufficient for a theoretical description of single-mode lasing in mode $h$ [35].

The second term of the master equation (2) captures the intermode kinetics and reads

$$
\begin{aligned}
C_{\mathrm{kin}}(\rho)= & -\sum_{i, j} R_{i \rightarrow j}\left[n_{i}\left(n_{j}+s\right) \rho_{N}^{n}\right. \\
& \left.-\left(n_{i}+1\right)\left(n_{j}-1+s\right) \rho_{N}^{\boldsymbol{n}+\mathbf{e}_{\mathrm{i}}-\mathbf{e}_{\mathrm{j}}}\right] .
\end{aligned}
$$

It is characterized by the rates $R_{i \rightarrow j}$ for a transition from mode $i$ to mode $j$. The rate asymmetry of the direct intermode transitions

$$
A_{i \rightarrow j}=R_{i \rightarrow j}-R_{j \rightarrow i}
$$

is generally nonzero. The origin of this rate asymmetry was attributed to stimulated scattering due to carrier population oscillations, e.g., in coupled photonic crystal nanolasers [18]. Furthermore, asymmetric backscattering of electromagnetic waves was observed in optical microcavities [58-60]. In our system, the rate asymmetry $A_{h \rightarrow l}$ is positive,

$$
A_{h \rightarrow l}>0,
$$

so the low-effective-gain mode $l$ is favored by the intermode kinetics. The parameter $s$ in Eq. (5) quantifies the ratio between spontaneous and induced intermode transitions. Its natural value is $s=1$. However, a reduction of $s$ to values $s<1$ is a simple way to effectively capture intermode interactions that lead to a relative enhancement of transitions into strongly occupied modes. We will show below that, while $s$ has (practically) no impact on the phase transition and the mean occupation(s) of the selected mode (s), it does affect the occupation of the nonselected mode. Only for $s<1$ does the master equation describe the experimentally observed superthermal fluctuations $g_{i l}^{(2)}>2$ of the nonselected mode. The numerical data shown in Figs. 2, 4, and 5 are obtained for $s=0$.

The form of the term $C_{\text {kin }}(\rho)$ capturing the intermode kinetics is identical to that of the master equation for an ideal gas of massive bosons in contact with an environment [41]. If such a system of massive bosons is coupled to a thermal environment characterized by the temperature $T$, the intermode rates obey $R_{j \rightarrow i} / R_{i \rightarrow j}=\exp \left[-\Delta_{i j} /\left(k_{B} T\right)\right]$ with Boltzmann constant $k_{B}$ and energy splitting $\Delta_{i j}=$ $E_{i}-E_{j}$ between modes (single-particle states) with energy $E_{i}$. In the quantum degenerate regime of low temperature or high boson density, the system will form a Bose-Einstein condensate in the single-particle state of lowest energy. When increasing the total number of bosons $N_{B}$ in this regime, the occupation of an excited mode $i$ approaches the finite value $\left\langle\hat{n}_{i}\right\rangle=\left(e^{E_{i} / k_{B} T}-1\right)^{-1}$, while the ground-state occupation increases linearly with $N_{B}$. Even for a finite number of discrete energy levels $i$, in the limit $N_{B} \rightarrow \infty$, this behavior clearly describes BoseEinstein condensation as the macroscopic occupation of one single-particle state (see, e.g., Ref. [42]).

The most intriguing result of this paper, shown below, is that the behavior of the bimodal system in the limit of strong pumping strength $P$ closely resembles that of a Bose-Einstein condensed gas of massive bosons in equilibrium. Remarkably, here the selected mode does not depend on the effective gain but is determined exclusively by the intermode transitions $R_{i \rightarrow j}$. This counterintuitive result is related to the fact that the intermode kinetics scales quadratically, but gain and loss scale only linearly with the mode occupations. 
It is instructive to define the dimensionless parameter $\varepsilon$,

$$
\frac{R_{l \rightarrow h}}{R_{h \rightarrow l}} \equiv \exp (-\varepsilon)
$$

which can be interpreted as the ratio $\varepsilon=\Delta_{h l}^{\text {eff }} /\left(k_{B} T_{\text {eff }}\right)$ of an effective energy splitting $\Delta_{h l}^{\text {eff }}$ between both modes and an effective temperature $T_{\text {eff }}$. In the limit of strong pumping, the intermode kinetics makes the photons condense into the mode corresponding to the lower effective energy. In other words, for $\varepsilon>0$ or $A_{h \rightarrow l}>0\left(\varepsilon<0\right.$ or $\left.A_{h \rightarrow l}<0\right)$, a Bose-Einstein condensate of photons is formed in mode $l$ (mode $h$ ). In contrast, it is always the mode $h$, characterized by the higher effective gain, that starts lasing when the pump power $P$ is ramped up. Thus, a rate asymmetry $A_{h \rightarrow l}>0$ implies a switching from lasing in mode $h$ to condensation in mode $l$, when the pump power is ramped up. In the following section, we derive an analytical theory, which describes this effect and establishes the analogy to equilibrium Bose-Einstein condensation for strong pumping.

\section{KINETIC THEORY}

\section{A. Mean-field approximation}

In order to obtain a closed set of kinetic equations for the mean mode occupations $\left\langle\hat{n}_{i}\right\rangle=\sum_{n, N} \rho_{N}^{n} n_{i}$ and the mean number of excited emitters $\langle\hat{N}\rangle=\sum_{n, N} \rho_{N}^{n} N$, we perform the mean-field approximation

$$
\left\langle\hat{n}_{i} \hat{n}_{j}\right\rangle \approx\left\langle\hat{n}_{i}\right\rangle\left\langle\hat{n}_{j}\right\rangle \quad \text { and }\left\langle\hat{N} \hat{n}_{i}\right\rangle \approx\langle\hat{N}\rangle\left\langle\hat{n}_{i}\right\rangle .
$$

This approximation, which ignores nontrivial twoparticle correlations, is later justified by comparing it to exact solutions of the full master equation (2) obtained from Monte Carlo simulations (see Fig. 2). Employing it, we derive kinetic equations of motion for the mean occupations:

$$
\begin{gathered}
\frac{d}{d t}\left\langle\hat{n}_{i}\right\rangle=\sum_{j}\left[R_{j \rightarrow i}\left\langle\hat{n}_{j}\right\rangle\left(\left\langle\hat{n}_{i}\right\rangle+s\right)-R_{i \rightarrow j}\left\langle\hat{n}_{i}\right\rangle\left(\left\langle\hat{n}_{j}\right\rangle+s\right)\right] \\
+g_{i}\langle\hat{N}\rangle\left(\left\langle\hat{n}_{i}\right\rangle+1\right)-\ell_{i}\left\langle\hat{n}_{i}\right\rangle \\
\frac{d}{d t}\langle\hat{N}\rangle=-\frac{\langle\hat{N}\rangle}{\tau}+P-\sum_{i} g_{i}\langle\hat{N}\rangle\left(\left\langle\hat{n}_{i}\right\rangle+1\right)
\end{gathered}
$$

\section{B. Asymptotic theory}

In a next step, for the sake of finding an analytical expression for the mean occupation(s) of the selected mode (s), in Eq. (10) we neglect spontaneous processes relative to corresponding stimulated ones, $\left(\left\langle\hat{n}_{i}\right\rangle+a\right) \simeq\left\langle\hat{n}_{i}\right\rangle$ with $a=1, s$. This approximation is valid asymptotically in the limit of large occupations of the emitters and the selected mode(s). Note, for high- $\beta$ cavities, where almost the entire spontaneous emission goes into the cavity modes, this assumption is plagued by the strong presence of spontaneous emission at the first threshold. Still, this assumption is valid in the coherent emission regime and, in particular, when describing transitions, where the selected modes change. Using the asymptotic approximation above, the stationary solution of Eq. (10) obeys

$$
\left\langle\hat{n}_{i}\right\rangle\left[g_{i}\langle\hat{N}\rangle-\ell_{i}+\sum_{j} A_{j \rightarrow i}\left\langle\hat{n}_{j}\right\rangle\right]=0 .
$$

This equation is solved by mean occupations that can be divided into two classes. For the nonselected modes $i \notin \mathcal{S}$, one finds the trivial solution $\left\langle\hat{n}_{i}\right\rangle=0$, whereas the occupations of the selected modes $i \in \mathcal{S}$ obey a linear set of equations:

$$
\sum_{j \in \mathcal{S}} A_{j \rightarrow i}\left\langle\hat{n}_{j}\right\rangle=\ell_{i}-g_{i}\langle\hat{N}\rangle, \quad \forall i \in \mathcal{S} .
$$

The occupation of the nonselected states, which vanishes in leading order [Eq. (12)], can be computed in the next order of our approximation. For this purpose, we take into account those terms that are linear in the number of excited emitters $\langle\hat{N}\rangle$ or the occupations $\left\langle\hat{n}_{i}\right\rangle$ of the selected modes. We find

$$
\left\langle\hat{n}_{i}\right\rangle=\frac{g_{i}\langle\hat{N}\rangle+s \sum_{j \in \mathcal{S}} R_{j \rightarrow i}\left\langle\hat{n}_{j}\right\rangle}{\ell_{i}-g_{i}\langle\hat{N}\rangle-\sum_{j \in \mathcal{S}} A_{j \rightarrow i}\left\langle\hat{n}_{j}\right\rangle} \quad \forall i \notin \mathcal{S} .
$$

The dependence of the number of excited emitters on the pumping can be obtained by analogical reasoning,

$$
\langle\hat{N}\rangle=\frac{P}{\tau^{-1}+\sum_{j \in \mathcal{S}} g_{j}\left\langle\hat{n}_{j}\right\rangle} .
$$

Following the strategy recently employed for massive bosons [41], the set of selected modes $\mathcal{S}$ can now be determined by the physical requirement that all modes (both selected and nonselected modes) must have positive occupations,

$$
\left\langle\hat{n}_{i}\right\rangle \geq 0 \quad \forall i .
$$

For the nonselected modes, this implies that the denominator of Eq. (14) has to be positive. Thus, the set of selected modes and their occupations are independent of the parameter $s$ since $s$ occurs neither in Eq. (13) nor in the denominator of Eq. (14). 


\section{Phase diagram of the bimodal microcavity system}

Based on the asymptotic theory, we can now compute the nonequilibrium phase diagram. The concept of selected modes, which appeared naturally in the asymptotic theory, clearly separates the parameter space into different phases, where no modes, one mode, or both modes are selected. A transition, where one of the nonselected modes becomes selected and starts emitting coherent light, is indicated by the divergence of its occupation described by Eq. (14). In turn, a selected mode ceases to be selected when its occupation, obtained by solving Eq. (13), drops to zero. In this section, we use this argument to compute the phase boundaries analytically. The resulting phase diagram is depicted in Fig. 3.

In phase $\mathrm{A}$, for small pumping power $P$, neither mode is selected, $\mathcal{S}=\{\}$. According to Eq. (14), the mode occupations read

$$
\left\langle\hat{n}_{i}\right\rangle_{\mathrm{A}} \approx \frac{1}{\ell_{i} /\left(g_{i}\langle\hat{N}\rangle\right)-1},
$$

and the number of excited emitters increases linearly with the pump rate,

$$
\langle\hat{N}\rangle_{\mathrm{A}}=\tau P .
$$

When the pumping is increased and reaches

$$
P_{\mathrm{AB}} \approx \frac{\ell_{h}}{g_{h} \tau},
$$

the occupation of the high-effective-gain mode diverges, indicating the transition to a regime where this mode is selected and starts emitting coherently [see Eq. (14)]. Since before the transition no mode is selected, this asymptotic estimate for the critical pumping strength cannot be expected to precisely mark the threshold in the high- $\beta$ limit. Note, however, that the estimates for further thresholds at $P_{\mathrm{BC}}$ and $P_{\mathrm{CD}}$ are not plagued by this problem since they occur in the regime where at least one mode is selected already.

In phase B, the high-effective-gain mode is selected, $\mathcal{S}=\{h\}$, and the number of excited emitters is clamped at the threshold value [see Eq. (13) and Fig. 2(a)]

$$
\langle\hat{N}\rangle_{\mathrm{B}}=\frac{\ell_{h}}{g_{h}} .
$$

The excitation provided by increasing the pumping is directly transferred to the selected mode [61]. Consequently, the occupation of the selected higheffective-gain mode depends linearly on the pump rate [use Eq. (15)],

$$
\left\langle\hat{n}_{h}\right\rangle_{\mathrm{B}}=\frac{P}{\ell_{h}}-\frac{1}{\tau g_{h}} .
$$

The occupation in the nonselected low-effective-gain mode is given by [see Eq. (14)]

$$
\left\langle\hat{n}_{l}\right\rangle_{\mathrm{B}}=\frac{g_{l}\langle\hat{N}\rangle_{\mathrm{B}}+s R_{h \rightarrow l}\left\langle\hat{n}_{h}\right\rangle_{\mathrm{B}}}{\ell_{l}-g_{l}\langle\hat{N}\rangle_{\mathrm{B}}-A_{h \rightarrow l}\left\langle\hat{n}_{h}\right\rangle_{\mathrm{B}}} .
$$

In a case where the mode-coupling rates favors the high-effective-gain mode $\left(A_{h \rightarrow l}<0\right)$, Eq. (22) would be valid for all pumping powers $P>P_{\mathrm{AB}}$. However, in our case, where the mode-coupling rates favor the loweffective-gain mode $\left(A_{h \rightarrow l}>0\right)$, increasing the pump rate (and, with it, also $\left\langle\hat{n}_{h}\right\rangle_{\mathrm{B}}$ ) eventually leads to the divergence of the right-hand side of Eq. (22). This occurs at the pump rate

$$
P_{\mathrm{BC}}=\frac{\ell_{h}}{g_{h}}\left[\frac{1}{\tau}+\frac{1}{A_{h \rightarrow l}}\left(g_{h} \ell_{l}-g_{l} \ell_{h}\right)\right]
$$

and indicates the transition to phase $\mathrm{C}$.

In phase $\mathrm{C}$, both modes are selected, $\mathcal{S}=\{h, l\}$. The number of excited emitters increases again linearly with the pump rate $P$,

$$
\langle\hat{N}\rangle_{\mathrm{C}}=P\left[\frac{1}{\tau}+\frac{1}{A_{h \rightarrow l}}\left(g_{h} \ell_{l}-g_{l} \ell_{h}\right)\right]^{-1} .
$$

The occupations of the high- and low-effective-gain mode decrease and increase linearly with $\langle\hat{N}\rangle_{\mathrm{C}}$, respectively,

$$
\begin{aligned}
\left\langle\hat{n}_{h}\right\rangle_{\mathrm{C}} & =-\frac{g_{l}\langle\hat{N}\rangle_{\mathrm{C}}-\ell_{l}}{A_{h \rightarrow l}}, \\
\left\langle\hat{n}_{l}\right\rangle_{\mathrm{C}} & =\frac{g_{h}\langle\hat{N}\rangle_{\mathrm{C}}-\ell_{h}}{A_{h \rightarrow l}} .
\end{aligned}
$$

When the number of emitters $\langle\hat{N}\rangle_{\mathrm{C}}$ reaches $\ell_{l} / g_{l}$, the occupation $\left\langle\hat{n}_{h}\right\rangle_{\mathrm{C}}$ becomes zero, indicating the transition to the phase where this mode is no longer selected. The threshold pump rate $P_{\mathrm{CD}}$ can be obtained from Eq. (24), analogously to the expression for $P_{\mathrm{BC}}$, and reads

$$
P_{\mathrm{CD}}=\frac{P_{\mathrm{BC}}}{G} .
$$

Thus, the extent of phase $\mathrm{C}$ is determined by the inverse effective-gain ratio $G$ [Eq. (4)].

In phase $\mathrm{D}$, only mode $l$ is selected, $\mathcal{S}=\{l\}$. The number of excited emitters remains at the higher threshold value,

$$
\langle\hat{N}\rangle_{\mathrm{D}}=\frac{\ell_{l}}{g_{l}},
$$


and the occupation of the selected mode $l$ reads

$$
\left\langle\hat{n}_{l}\right\rangle_{\mathrm{D}}=\frac{P}{\ell_{l}}-\frac{1}{\tau g_{l}} .
$$

The occupation of the nonselected mode $h$ is given by

$$
\left\langle\hat{n}_{h}\right\rangle_{\mathrm{D}}=\frac{g_{h}\langle\hat{N}\rangle_{\mathrm{D}}+s R_{l \rightarrow h}\left\langle\hat{n}_{l}\right\rangle_{\mathrm{D}}}{\ell_{h}-g_{h}\langle\hat{N}\rangle_{\mathrm{D}}+A_{h \rightarrow l}\left\langle\hat{n}_{l}\right\rangle_{\mathrm{D}}} .
$$

The crucial difference from Eq. (22) is that for $A_{h \rightarrow l}>0$, an increase of $\left\langle\hat{n}_{l}\right\rangle_{\mathrm{D}}$ cannot produce a further root in the denominator of Eq. (30). Thus, no further transition will occur (unless other parameters change as well when the pump power is ramped up).

Figure 3 shows the phase diagram resulting from the asymptotic theory with respect to the effective-gain ratio $G$ (varied by varying the gain rate $g_{l}$ ) and the pumping strength $P$ (proportional to the injection current $I$ ). While the precise shape of the phase boundaries depends on the parameters (and which of them are varied in order to modify the effective-gain ratio $G$ ), the topology of the phase diagram is generic. For $G<1$ (and $A_{h \rightarrow l}>0$ ), the system always undergoes a sequence of three transitions between the phases A, B, C, and D when the pump rate is increased. For $G>1$ (and $A_{h \rightarrow l}>0$ ), where the mode labeled $l$ actually becomes the high-effective-gain mode, only a single transition from phase A to phase D occurs. In summary, when the pump power $P$ is ramped up, the system starts lasing in the mode characterized by the higher effective gain, whereas in the limit of strong pumping, the selected mode is the one favored by the intermode kinetics. Moreover, the switching from selection of mode $h$ to selection of mode $l$ has to occur via an intermediate phase, where both modes are selected (unless the system is fine-tuned to $G=1$ ).

The data plotted in Fig. 2 corresponds to a cut through this phase diagram following the dashed horizontal line in Fig. 3. The different phases obtained from the asymptotic theory are indicated by the colors at the bottom of panel (a). In Fig. 2(a), we can clearly see that the mean occupations obtained from the asymptotic theory (dashed lines) nicely reproduce the exact solution (solid line) of the master equation (2), which was obtained by Monte Carlo simulations (for a detailed description of the method, see Ref. [42]). This agreement justifies both the mean-field approximation and the asymptotic theory. More importantly, the theoretical curves also describe the experimental data (circles in Fig. 2) very well. In Appendix A, we explain how the parameters of our model are determined.

\section{Relation to Bose-Einstein condensation}

We have seen that in the limit of strong pumping strength $P$, the selected mode is determined exclusively by the intermode kinetics, which is described by the rates $R_{i \rightarrow j}$. It is remarkable that in this limit, neither the loss rates of the modes $\ell_{i}$ nor their coupling $g_{i}$ to the emitters influence the selection of the mode. This counterintuitive result is related to the fact that gain and loss scale only linearly with the mode occupations, whereas the rates for the intermode kinetics have a quadratic dependence on the mode occupations. This implies that the mechanism leading to a macroscopic (or large) occupation of one of the modes is the same as the one that leads to the Bose-Einstein condensation of massive bosons in contact with a thermal environment. This result is based on the Bose-enhanced inter-mode kinetics (scattering) described, e.g., in Ref. [41] on the basis of a rate equation comprising the same terms as $C_{\text {kin }}$ [Eq. (5)].

Even though the system consists of two levels only, the notion of Bose condensation becomes sharp in the limit $P \rightarrow \infty$, where the occupation of mode $l$, given by Eq. (29), approaches infinity, while that of mode $h$, given by Eq. (30), remains below a finite value. Note that the number of excited emitters, given by Eq. (28), also remains at a finite value in this limit. This result has the important consequence that the occupation of the noncondensed mode $h$ is determined completely by the intermode kinetics described by the rates $R_{i \rightarrow j}$. In the limit $P \rightarrow \infty$, it approaches

$$
\left\langle\hat{n}_{h}\right\rangle \simeq \frac{s}{\exp (\varepsilon)-1},
$$

where $\varepsilon$ is the parameter defined in Eq. (8). Thus, for $s=1$, the occupation of mode $l$ precisely corresponds to that of an excited state of energy $\varepsilon k_{B} T$ in a Bose condensed system of massive bosons at temperature $T$. The fact that the "excitedstate" occupation $\left\langle\hat{n}_{h}\right\rangle$ (the depletion) approaches a constant value for strong pumping, so that increasing the number of photons (bosons) in the system will only increase the condensate occupation, is another clear analogy to equilibrium Bose condensation. Irrespective of the value of $s$, for strong pumping, the selection of the coherent emission mode is a result of the intermode kinetics.

\section{PHOTON STATISTICS}

In microlasers, where almost the entire spontaneous emission goes into a single mode, no sharp intensity jump is visible at the lasing threshold [5,35]. Instead, the transition to coherent emission is indicated in the autocorrelation [62] of the emitted photons [57,63-68]. To confirm the coherence properties of the emitted photons, we examine the photon statistics. The equal-time photon correlation functions (1), which we can write like

$$
g_{i j}^{(2)}= \begin{cases}\left(\left\langle\hat{n}_{i} \hat{n}_{i}\right\rangle-\left\langle\hat{n}_{i}\right\rangle\right) /\left\langle\hat{n}_{i}\right\rangle^{2} & \text { if } i=j \\ \left\langle\hat{n}_{i} \hat{n}_{j}\right\rangle /\left\langle\hat{n}_{i}\right\rangle\left\langle\hat{n}_{j}\right\rangle & \text { if } i \neq j,\end{cases}
$$

measure the occupation number fluctuations of each mode and the cross-correlation between the modes, respectively. 


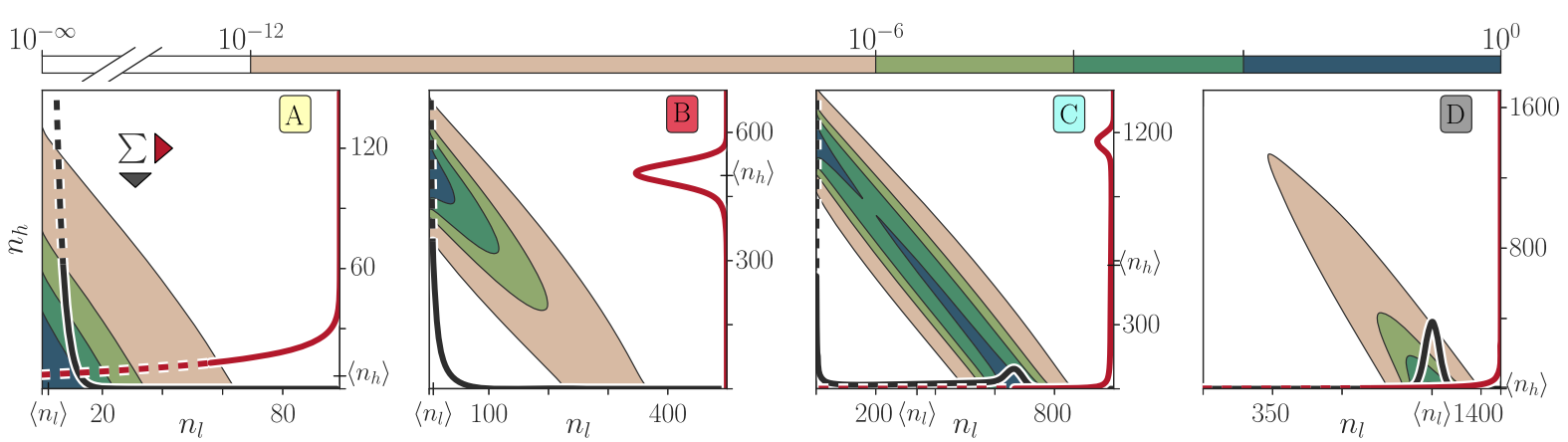

FIG. 4. Two-mode photon statistics obtained from the reduced master equation (see Appendix B) with single-mode statistics for mode $l$ at the bottom (black) and mode $h$ at the right (red) axis, for injection currents $I=12.5 \mu \mathrm{A}, 27.4 \mu \mathrm{A}, 41.7 \mu \mathrm{A}, 60.0 \mu \mathrm{A}(\mathrm{phases} \mathrm{A}, \mathrm{B}$, C, D). These pump rates are also indicated by arrows in Fig. 2(b). The labels $\left\langle\hat{n}_{h}\right\rangle$ and $\left\langle\hat{n}_{l}\right\rangle$ mark the position of the expectation value for each mode. For a better comparison, the single-mode statistics are depicted up to $0.025 \%$ by solid lines, while the remaining part is shown by dashed lines. Note that the plotted range of photon numbers increases from left to right.

In Figs. 2(b) and 2(c), experimental and theoretical results for $g_{i i}^{(2)}$ and $g_{h l}^{(2)}$ are depicted. The theoretical values for $g_{i j}^{(2)}$ are determined by a numerically exact Monte Carlo simulation of the full master equation (2). As discussed above, a change from $g_{h h}^{(2)}=2$ to $g_{h h}^{(2)}=1$ indicates the first threshold where the coherent emission in mode $h$ sets in, as can be seen clearly in Fig. 2. For even stronger pumping, when entering or leaving phase $C$, in which both modes are selected, we observe pronounced anticorrelations between both modes $\left(g_{h l}<1\right)$, as well as superthermal intensity fluctuations $\left(g_{i i}>2\right)$ of mode $i$ that changes its state from nonselected to selected or vice versa. Note that for our system parameters, phase $\mathrm{C}$ appears in a narrow interval of pump powers only, so its properties are overshadowed by those of the transitions $\mathrm{BC}$ and $\mathrm{CD}$. As a result, the two minima of the cross-correlations occurring at the transitions have merged to a single one.

In order to reproduce the measured superthermal intensity fluctuations at the transitions BC and CD theoretically, we have to choose $s<1$, corresponding to the presence of intermode interactions. The best results are obtained for the value $s=0$, which we also used in the simulations (the role of $s$ will be discussed in more detail below and in Appendix C).

We now investigate the signatures of the mode switching in the reduced two-mode photon distribution $\rho^{n_{h}, n_{l}}=$ $\sum_{N} \rho_{N}^{n_{h}, n_{l}}$, which gives the probability to find the system in a state with $n_{h}$ and $n_{l}$ photons in mode $h$ and mode $l$, respectively. We compute this quantity by solving either the full master equation (phases A and B) or the reduced master equation for $\rho^{n_{h}, n_{l}}$ (phases $\mathrm{C}$ and $\mathrm{D}$, see Appendix B for details). Results for four different pump powers $P$, corresponding to the four phases A to D, are depicted in Fig. 4. The corresponding single-mode distributions $\rho^{n_{h}}=\sum_{n_{l}} \rho^{n_{h}, n_{l}}$ and $\rho^{n_{l}}$ are shown as well.

In the nonselected phase $\mathrm{A}$, the distribution possesses a single maximum at $\boldsymbol{n}=\left(n_{h}, n_{l}\right)=(0,0)$. The selection of mode $h$ (phases B and C) is associated with a maximum of the distribution at $\boldsymbol{n}=\left(n_{h}^{\max }, 0\right)$ lying on the vertical axis, whereas the selection of mode $l$ (phases C and D) is associated with a maximum at $\boldsymbol{n}=\left(0, n_{l}^{\max }\right)$ lying on the horizontal axis. Thus, in phase $\mathrm{C}$, where both modes are selected, the distribution possesses two local maxima that are separated by a saddle point [69]. The emergence of the second maximum when entering phase $\mathrm{C}$ is also visible in the occupation distribution of the mode that starts emitting coherently, as can be seen in Fig. 5, showing $\rho^{n_{l}}$ directly before and after the transition from B to $\mathrm{C}$. The buildup and, later, the presence of the second maximum in $\rho^{n_{l}}$ are accompanied by the strong (superthermal) number fluctuations in this mode.

The impact of the effective parameter $s$ is illustrated in Fig. 6, where the two-mode distribution is shown for $s=1$ in phases $\mathrm{B}$ and $\mathrm{C}$ for the same parameters used in the corresponding panels in Fig. 4. For $s=1$, states with zero occupation in one of the modes (situated along the axes of the plot) are much less attractive than for the value $s=0$ considered before. As a striking consequence, the selection of both modes in phase $\mathrm{C}$ is not associated with two
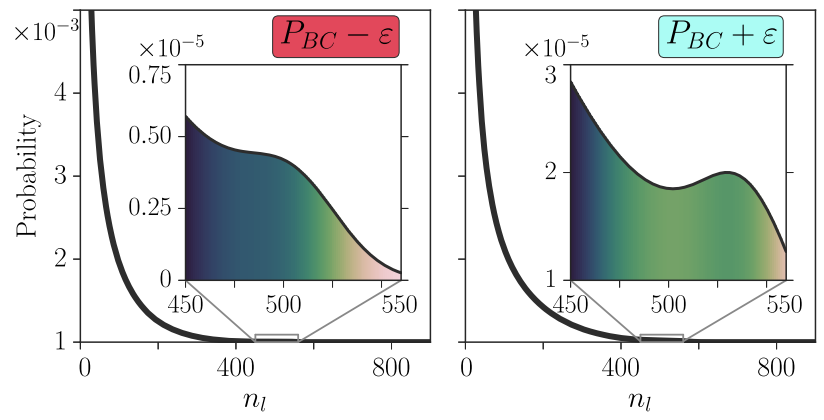

FIG. 5. Photon statistics of mode $l\left(\rho^{n_{l}}\right)$ for pump rates below $\left(P_{B C}-\varepsilon\right.$, left panel) and above $\left(P_{B C}+\varepsilon\right.$, right panel $)$ with $\varepsilon=0.016 \mu \mathrm{A}$. Only in the right panel the photon statistics exhibits a second local maximum. The system parameters are the same as in Fig. 2. 

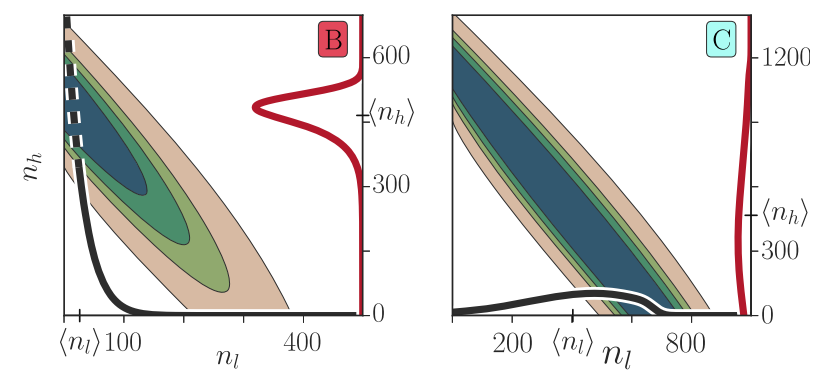

FIG. 6. Two-mode photon statistics obtained from the reduced master equation for the same parameters as in Fig. 4, B and C, but with spontaneous transitions fully included between the modes. In contrast to the case with suppressed spontaneous transitions, the statistics are no longer attracted to the axis. The color bar is the same as in Fig. 4. For a better comparison, the single-mode statistics are depicted up to $0.015 \%$ by solid lines, while the remaining part is shown by dashed lines.

maxima in the distribution anymore, but rather with a single central maximum. The transition from phase $\mathrm{B}$ to phase $\mathrm{C}$ now corresponds to the shifting of the single maximum away from the horizontal axis. As a result, for $s=1$, the system does not show the experimentally observed superthermal photon number fluctuations, as we discuss in more detail in Appendix C. It is an interesting observation that neglecting or taking into account the spontaneous emission between the modes has such a strong impact on the statistical properties of the modeled bimodal system. Note, however, that despite this strong impact on the statistics, the mean occupations of the modes and the critical parameters for the phase transition do not show a strong dependence on $s$. This is also the result predicted by the asymptotic theory presented in the previous section.

\section{CONCLUSION}

In this paper, we investigated the pump-power-driven mode switching in a bimodal microcavity. We presented experimental results and their explanation in terms of a transparent analytical theory. In particular, we found that the transition has to occur via an intermediate phase where both modes are selected. Our theoretical description reveals, moreover, a close connection to the physics of equilibrium Bose-Einstein condensation in quantum gases of massive bosons. The mode switching can, therefore, be viewed as a minimal instance of Bose-Einstein condensation of photons and its demarcation to lasing.

We also investigated the statistical properties of the system and pointed out that the mode switching is accompanied by superthermal intensity fluctuations as well as anticorrelations between both modes. This observation can be technically relevant since a device producing a drastically increased occurrence rate of photon pairs (with a very narrow linewidth [45]) could be used to enhance two-photon excitation processes used in fluorescence microscopy [70]. Furthermore, a device that changes its predominantly emitting mode in dependence of the injection current could be applied for optical memories and other types of mode management [12,71].

\section{ACKNOWLEDGMENTS}

The authors thank A. Musiał for very helpful comments on the manuscript. T. L., D. V., and H. A. M. L. contributed equally to this work. D. V. is grateful for support from the Studienstiftung des Deutschen Volkes. We acknowledge funding from the European Research Council under the European Union's Seventh Framework ERC Grant Agreement No. 615613 and from the German Research Foundation (DFG) via Project No. Re2974/3-1 and the Research Unit FOR2414.

\section{APPENDIX A: EXTRACTING SYSTEM PARAMETERS FROM THE MEASURED DATA}

The asymptotic theory describes the generic form of the mode switching, and its analytic expressions can thus be used to obtain the parameters of the master equation model. However, the theoretical parameters cannot be related directly to experimental parameters because of the unknown proportionality factor $a_{i}$ between the intensity of the emitted light $J_{i}$ and the occupation of modes, $\left\langle\hat{n}_{i}\right\rangle=a_{i} J_{i}$, and the unknown excitation efficiency $b$ of the pumping with respect to the injection current, $P=b I$.

The main properties of the switching are captured by the effective-gain ratio $G$, which determines whether a switching occurs, and the extent of phase $\mathrm{C},\left(P_{\mathrm{CD}} / P_{\mathrm{BC}}\right)=G^{-1}$ [see Eq. (27)]. This ratio can be obtained in the following way: First, apply a linear fit for the intensities of the selected modes $i$ in each phase $R \in\{\mathrm{B}, \mathrm{C}, \mathrm{D}\}$

$$
\left.J_{i}(I)\right|_{R}=A_{i R} I+B_{i R} .
$$

The ratio $\left(P_{\mathrm{CD}} / P_{\mathrm{BC}}\right)=\left(I_{\mathrm{CD}} / I_{\mathrm{BC}}\right)$ is then determined either by the intersections of $\left.J_{h}\right|_{\mathrm{B}}\left(I_{\mathrm{BC}}\right)=\left.J_{h}\right|_{\mathrm{C}}\left(I_{\mathrm{BC}}\right)$ and $\left.J_{l}\right|_{\mathrm{C}}\left(I_{\mathrm{CD}}\right)=\left.J_{l}\right|_{\mathrm{D}}\left(I_{\mathrm{CD}}\right)$ or via the points where the occupation of the modes approach zero, $\left.J_{h}\right|_{\mathrm{C}}\left(I_{\mathrm{CD}}\right)=0$ and $\left.J_{l}\right|_{\mathrm{C}}\left(I_{\mathrm{BC}}\right)=0$. Both procedures give similar values for the effective-gain ratio $G$ via Eq. (27), namely, 1.22 and 1.27, respectively. Thus, determining $G$ does not require the knowledge of the excitation efficiency $b$ and the absolute number of cavity photons via $a_{i}$.

The parameters $A_{h \rightarrow l}, g_{h}, g_{l}, \ell_{h}$, and $\ell_{l}$, are extracted for comparison between theory and experiment via the leastsquares method for all experimental data with $I<80 \mu \mathrm{A}$ and are listed in the caption of Fig. 2. Since the time scale in Eq. (2) does not affect steady-state properties, all parameters are measured in units of $\tau$. The individual rates $R_{l \rightarrow h}$ and $R_{h \rightarrow l}$ do not affect the asymptotic theory; only the rate asymmetry $A_{l \rightarrow h}$ does (as discussed above). But the correlation function $g_{l l}^{(2)}$ does depend on the 
individual rates, so $R_{l \rightarrow h}$ is chosen to reproduce this correlation function.

In the experiment, the orientation of a polarization filter is chosen parallel to the passive cavity modes at the inversion point. Because of the interactions induced by the common gain medium, the polarization-resolved spectrum exhibits a double-peak structure, indicating that each polarization direction contains small portions of the other mode [45]. To make numerical and experimental fluctuations comparable, we take into account that in each polarization a small fraction of the other mode is detected by introducing the mixing

$$
n_{h, l}^{\text {meas }}=(1-c) n_{h, l} \pm c n_{l, h},
$$

where $n_{h, l}^{\text {meas }}$ denotes the quantity that is measured. The mode mixing prevents the experimental observed fluctuations of the nonselected mode from increasing monotonically in phase D [see Fig. 2(b)]. Comparing the theoretical results with the experimental data, we find the optimal value of the mixing parameter to be very small, $c=8 \times 10^{-3}$. Its impact of the mixing is negligible in phases $\mathrm{A}, \mathrm{B}$, and $\mathrm{C}$. It is taken into account in the numerical data presented in Figs. 2 and 8, where the index "meas" is dropped.

\section{APPENDIX B: REDUCED DENSITY MATRIX}

Under the (idealizing) assumption that all spontaneous emission goes into the selected modes i.e., $\tau^{-1}=0$ and $\beta=1$, a reduced density matrix of the form $\rho^{n_{h}, n_{l}}$ can be derived. To derive the equation of motion for the reduced density matrix, we need to consider only the parts of Eq. (2) describing the pump and the photon emission into the cavity,

$$
\begin{aligned}
\frac{d}{d t} \rho_{N}^{n}= & +P\left[\rho_{N-1}^{n}-\rho_{N}^{n}\right]+[\ldots] \\
& -\sum_{i} g_{i}\left[N\left(n_{i}+1\right) \rho_{N}^{n}-(N+1) n_{i} \rho_{N+1}^{n-\mathbf{e}_{i}}\right] .
\end{aligned}
$$

Here and in the following equation, $[\ldots]$ stands for terms that describe the loss of the individual modes as well as intermode transitions. When no spontaneous emission is lost into nonselected modes, the carrier recombination and emission into the cavity modes is faster than any other process, so the term $\sum_{i} g_{i} N\left(n_{i}+1\right) \rho_{N}^{n}$ can be substituted by $P \rho_{N-1}^{n}$. This means that whenever an emitter is excited by the pump, its excitation is immediately emitted into the cavity; thus, the emission into the modes can be described directly by $P \rho_{N-1}^{n}$ [35]. By the same reasoning or by simply shifting the indices $\left(\rho_{N+1}^{n-\mathbf{e}_{\mathrm{i}}}\right)$, one can find a substitute for all terms in Eq. (2) that correspond to the photon emission. Now, we can trace over the emitter subspace $\left(\sum_{N}\right)$, resulting in an equation of motion for the reduced density matrix,

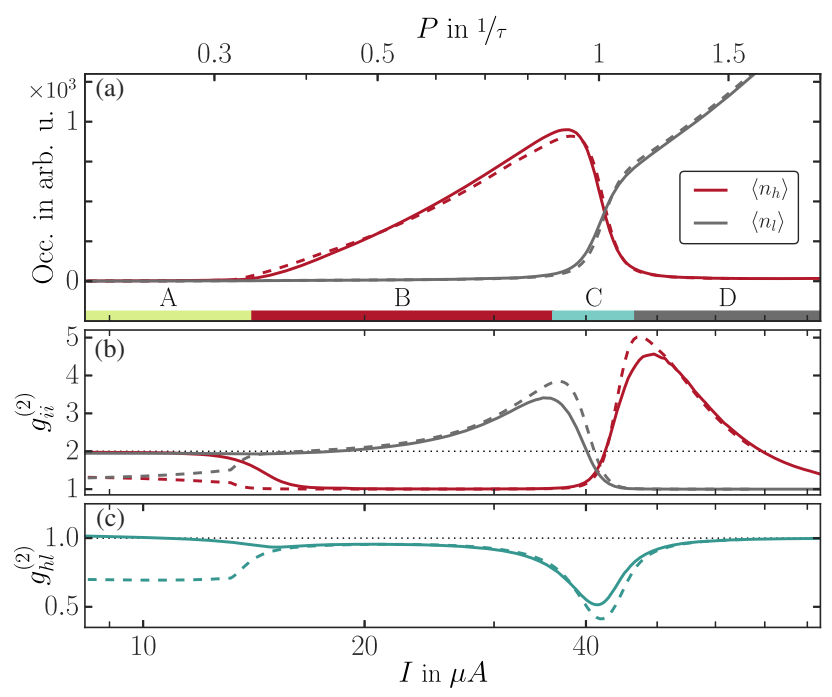

FIG. 7. Comparison of $\left\langle n_{i}\right\rangle$ and $g_{i j}^{(2)}$ obtained from the numerical, exact Monte Carlo simulation of Eq. (2) (solid lines) with the ones obtained from the direct solution of the reduced density matrix Eq. (B1) (dashed lines). Note that the pump in the reduced density matrix is scaled to compensate for $\beta \neq 1$.

$$
\frac{d}{d t} \rho^{n}=-P \rho^{n}+\sum_{i} \frac{P g_{i} n_{i} \rho^{n-\mathbf{e}_{\mathbf{i}}}}{\sum_{j} g_{j}\left(n_{j}+1\right)-g_{i}}+[\ldots]
$$

As argued above, the reduced density matrix approach works under the assumption of $\beta=1$. In our case, $\beta \simeq 0.2$, so only a fraction of the pump effectively creates photons in the cavity. To be able to compare the reduced density matrix to the full model, the pump power is scaled accordingly. Figure 7 shows the results of the Monte Carlo simulations of the full equation compared to the results obtained from the numerical solution of the reduced equation. The deviation of the reduced density matrix approach for small pump rates is not a problem since for low pump rates the full equation can still be solved numerically (as is done for Fig. 4, A and B). Importantly, the reduced equation reproduces the results in the regime of high pump rates, where the exact solution for the full equation can no longer be obtained.

\section{APPENDIX C: ROLE OF SPONTANEOUS INTERMODE TRANSITIONS}

In this appendix, we investigate the impact of the effective parameter $s$, which quantifies spontaneous intermode transitions. Figure 8 shows the mode characteristics for the case with full spontaneous transitions between the modes $(s=1)$. In contrast to Fig. 2, the sharp kinks in the occupations [panel (a)] and the cross-correlation [panel (c)] in phase $\mathrm{C}$ are less pronounced. However, the most significant deviation appears in the photon autocorrelations. For $s=1$, the computed autocorrelation does not 

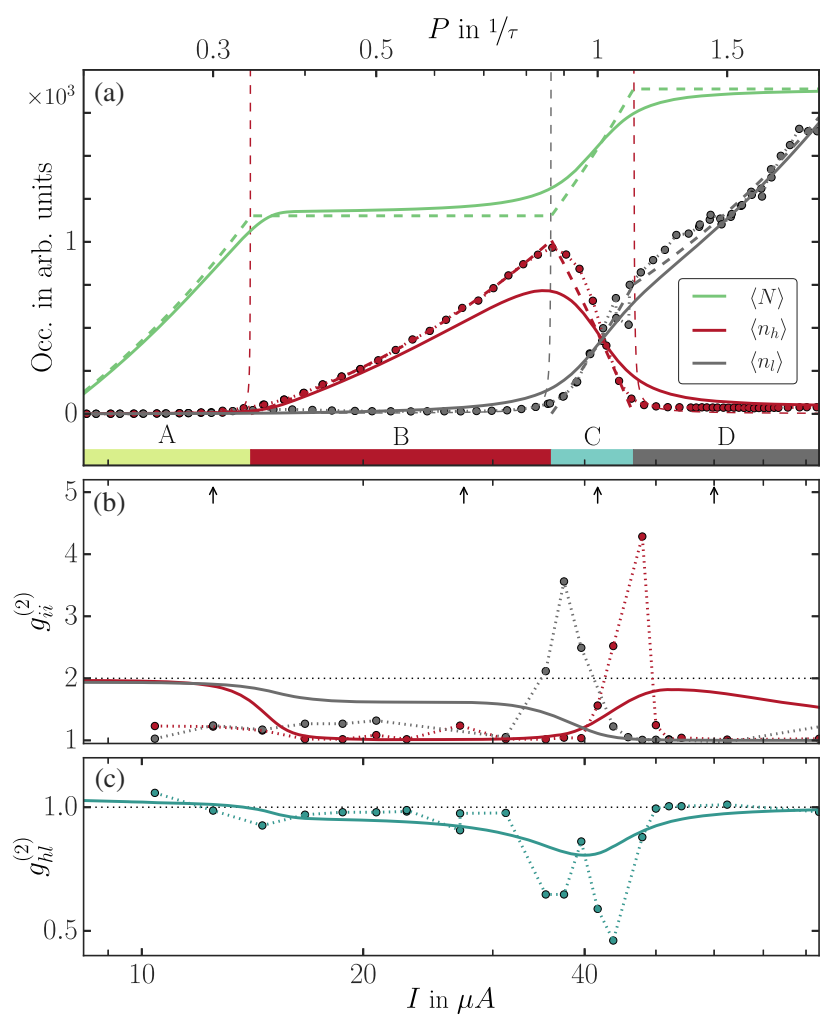

FIG. 8. Calculated input-output characteristics and fluctuation for both modes in the presence of spontaneous transitions between the modes, $s=1$. The fluctuations are restricted to values $g_{i i}^{(2)} \leq 2$. All parameters are chosen as in Fig. 2 .

reproduce the experimentally observed superthermal fluctuations.

This numerical observation can be backed up analytically using the following argument. In phases B and D, the joint distribution $\rho_{N}^{n}$ factorizes approximately into two parts, one describing the nonselected mode $i$ and the other one describing the selected mode $j$ and the emitters, $\rho_{N}^{n} \approx \rho^{n_{i}} \rho_{N}^{n_{j}}$. This statement is confirmed by the numerical solution of the full master equation. Such a factorization allows one to trace out both the selected mode and the emitters, to obtain an equation of motion for the photon number distribution of the nonselected mode:

$$
\begin{aligned}
\frac{d}{d t} \rho^{n}= & -\langle\hat{N}\rangle g\left[(n+1) \rho^{n}-n \rho^{n-1}\right] \\
& -\ell\left[n \rho^{n}-(n+1) \rho^{n+1}\right] \\
& -R_{j \rightarrow i}\left\langle\hat{n}_{j}\right\rangle\left[(n+s) \rho^{n}-(n-1+s) \rho^{n-1}\right] \\
& -R_{i \rightarrow j}\left(\left\langle\hat{n}_{j}\right\rangle+s\right)\left[n \rho^{n}-(n+1) \rho^{n+1}\right] .
\end{aligned}
$$

Here, $n$ denotes the photon number of the nonselected mode; $\left\langle\hat{n}_{j}\right\rangle,\langle\hat{N}\rangle$ the mean occupations of the selected mode and the emitters, respectively; $R_{j \rightarrow i}\left(R_{i \rightarrow j}\right)$ the transition rate from (to) the selected mode $j$ to (from) the nonselected mode; and $g, \ell$ the gain and loss rate of the nonselected mode. If spontaneous transitions are fully included, $s=1$, Eq. (C1) is solved by a distribution of the form $\rho^{n}=(1-\alpha) \alpha^{n}$, which always yields $g^{(2)}=2$. This explains why we do not find superthermal fluctuations for $s=1$.

[1] L. He, Ş. K. Özdemir, and L. Yang, Whispering Gallery Microcavity Lasers, Laser Photonics Rev. 7, 60 (2013).

[2] K. J. Vahala, Optical Microcavities, Nature (London) 424, 839 (2003).

[3] N. V. Kryzhanovskaya, M. V. Maximov, and A. E. Zhukov, Whispering-Gallery Mode Microcavity Quantum-Dot Lasers, Quantum Electron. 44, 189 (2014).

[4] H. Cao and J. Wiersig, Dielectric Microcavities: Model Systems for Wave Chaos and Non-Hermitian Physics, Rev. Mod. Phys. 87, 61 (2015).

[5] M. Lermer, N. Gregersen, M. Lorke, E. Schild, P. Gold, J. Mørk, C. Schneider, A. Forchel, S. Reitzenstein, S. Höfling, and M. Kamp, High Beta Lasing in Micropillar Cavities with Adiabatic Layer Design, Appl. Phys. Lett. 102, 052114 (2013).

[6] M. Nomura, N. Kumagai, S. Iwamoto, Y. Ota, and Y. Arakawa, Laser Oscillation in a Strongly Coupled SingleQuantum-Dot-Nanocavity System, Nat. Phys. 6, 279 (2010).

[7] P. Lett, W. Christian, S. Singh, and L. Mandel, Macroscopic Quantum Fluctuations and First-Order Phase Transition in a Laser, Phys. Rev. Lett. 47, 1892 (1981).

[8] L. Mandel and E. Wolf, Optical Coherence and Quantum Optics (Cambridge University Press, Cambridge, England, 1995).

[9] M. Sondermann, M. Weinkath, T. Ackemann, J. Mulet, and S. Balle, Two-Frequency Emission and Polarization Dynamics at Lasing Threshold in Vertical-Cavity SurfaceEmitting Lasers, Phys. Rev. A 68, 033822 (2003).

[10] H. A. M. Leymann, C. Hopfmann, F. Albert, A. Foerster, M. Khanbekyan, C. Schneider, S. Höfling, A. Forchel, M. Kamp, J. Wiersig, and S. Reitzenstein, Intensity Fluctuations in Bimodal Micropillar Lasers Enhanced by QuantumDot Gain Competition, Phys. Rev. A 87, 053819 (2013).

[11] H. A. M. Leymann, A. Foerster, M. Khanbekyan, and J. Wiersig, Strong Photon Bunching in a Quantum-Dot-Based Two-Mode Microcavity Laser, Phys. Status Solidi B 250, 1777 (2013).

[12] S. V. Zhukovsky, D. N. Chigrin, A. V. Lavrinenko, and J. Kroha, Switchable Lasing in Multimode Microcavities, Phys. Rev. Lett. 99, 073902 (2007).

[13] K. D. Choquette, D. A. Richie, and R.E. Leibenguth, Temperature Dependence of Gain-Guided Vertical-Cavity Surface Emitting Laser Polarization, Appl. Phys. Lett. 64, 2062 (1994).

[14] D. Sun, E. Towe, P. H. Ostdiek, J. W. Grantham, and G. J. Vansuch, Polarization Control of Vertical-Cavity SurfaceEmitting Lasers through Use of an Anisotropic Gain Distribution in [110]-Oriented Strained Quantum-Well Structures, IEEE J. Sel. Top. Quantum Electron. 1, 674 (1995). 
[15] J. Martin-Regalado, J. L. A. Chilla, J. J. Rocca, and P. Brusenbach, Polarization Switching in Vertical-Cavity Surface Emitting Lasers Observed at Constant Active Region Temperature, Appl. Phys. Lett. 70, 3350 (1997).

[16] J. Rubio, L. Pfeiffer, M. H. Szymanska, A. Pinczuk, S. He, H. U. Baranger, P. B. Littlewood, K. W. West, and B. S. Dennis, Coexistence of Excitonic Lasing with Electron-Hole Plasma Spontaneous Emission in One-Dimensional Semiconductor Structures, Solid State Commun. 120, 423 (2001).

[17] T. Ackemann and M. Sondermann, Characteristics of Polarization Switching from the Low to the High Frequency Mode in Vertical-Cavity Surface-Emitting Lasers, Appl. Phys. Lett. 78, 3574 (2001).

[18] M. Marconi, J. Javaloyes, F. Raineri, J. A. Levenson, and A. M. Yacomotti, Asymmetric Mode Scattering in Strongly Coupled Photonic Crystal Nanolasers, Opt. Lett. 41, 5628 (2016).

[19] L. Ge, D. Liu, A. Cerjan, S. Rotter, H. Cao, S. G. Johnson, H. E. Türeci, and A. Douglas Stone, Interaction-Induced Mode Switching in Steady-State Microlasers, Opt. Express 24, 41 (2016).

[20] S. S. Alharthi, A. Hurtado, V.-M. Korpijarvi, M. Guina, I. D. Henning, and M. J. Adams, Circular Polarization Switching and Bistability in an Optically Injected $1300 \mathrm{~nm}$ SpinVertical Cavity Surface Emitting Laser, Appl. Phys. Lett. 106, 021117 (2015).

[21] G. S. Agarwal and S. Dattagupta, Higher-Order Phase Transitions in Systems Far from Equilibrium: Multicritical Points in Two-Mode Lasers, Phys. Rev. A 26, 880 (1982).

[22] P. Gartner and C. M. Halati, Laser Transition in the Thermodynamic Limit for Identical Emitters in a Cavity, Phys. Rev. A 93, 013817 (2016).

[23] J.Kasprzak, M. Richard, S. Kundermann, A. Baas, P. Jeambrun, J. M. J. Keeling, F. M. Marchetti, M. H. Szymanska, R. Andre, J. L. Staehli, V. Savona, P. B. Littlewood, B. Deveaud, and L. S. Dang, Bose-Einstein Condensation of Exciton Polaritons, Nature (London) 443, 409 (2006).

[24] R. Balili, V. Hartwell, D. Snoke, L. Pfeiffer, and K. West, Bose-Einstein Condensation of Microcavity Polaritons in a Trap, Science 316, 1007 (2007).

[25] H. Deng, H. Haug, and Y. Yamamoto, Exciton-Polariton Bose-Einstein Condensation, Rev. Mod. Phys. 82, 1489 (2010).

[26] E. Wertz, L. Ferrier, D. D. Solnyshkov, R. Johne, D. Sanvitto, A. Lematre, I. Sagnes, R. Grousson, A. V. Kavokin, P. Senellart, G. Malpuech, and J. Bloch, Spontaneous Formation and Optical Manipulation of Extended Polariton Condensates, Nat. Phys. 6, 860 (2010).

[27] I. Carusotto and C. Ciuti, Quantum Fluids of Light, Rev. Mod. Phys. 85, 299 (2013).

[28] T. Byrnes, N. Y. Kim, and Y. Yamamoto, Exciton-Polariton Condensates, Nat. Phys. 10, 803 (2014).

[29] J. Fischer, I. G. Savenko, M. D. Fraser, S. Holzinger, S. Brodbeck, M. Kamp, I. A. Shelykh, C. Schneider, and S. Höfling, Spatial Coherence Properties of One Dimensional Exciton-Polariton Condensates, Phys. Rev. Lett. 113, 203902 (2014).

[30] S. O. Demokritov, V.E. Demidov, O. Dzyapko, G. A. Melkov, A. A. Serga, B. Hillebrands, and A. N. Slavin,
Bose-Einstein Condensation of Quasi-equilibrium Magnons at Room Temperature under Pumping, Nature (London) 443, 430 (2006).

[31] Y. M. Bunkov and G. E. Volovik, Bose-Einstein Condensation of Magnons in Superfluid ${ }^{3} \mathrm{He}$, J. Low Temp. Phys. 150, 135 (2008).

[32] O. Vainio, J. Ahokas, J. Järvinen, L. Lehtonen, S. Novotny, S. Sheludiakov, K.-A. Suominen, S. Vasiliev, D. Zvezdov, V. V. Khmelenko, and D. M. Lee, Bose-Einstein Condensation of Magnons in Atomic Hydrogen Gas, Phys. Rev. Lett. 114, 125304 (2015).

[33] F. Fang, R. Olf, S. Wu, H. Kadau, and D. M. Stamper-Kurn, Condensing Magnons in a Degenerate Ferromagnetic Spinor Bose Gas, Phys. Rev. Lett. 116, 095301 (2016).

[34] J. Klaers, J. Schmitt, F. Vewinger, and M. Weitz, BoseEinstein Condensation of Photons in an Optical Microcavity, Nature (London) 468, 545 (2010).

[35] P. R. Rice and H. J. Carmichael, Photon Statistics of a Cavity-QED Laser: A Comment on the Laser-PhaseTransition Analogy, Phys. Rev. A 50, 4318 (1994).

[36] J. Knebel, T. Krüger, M. F. Weber, and Erwin Frey, Coexistence and Survival in Conservative Lotka-Volterra Networks, Phys. Rev. Lett. 110, 168106 (2013).

[37] J. Knebel, M. F. Weber, T. Krueger, and E. Frey, Evolutionary Games of Condensates in Coupled Birth-Death Processes, Nat. Commun. 6, 6977 (2015), .

[38] O. Hirschberg, D. Mukamel, and G. M. Schütz, Condensation in Temporally Correlated Zero-Range Dynamics, Phys. Rev. Lett. 103, 090602 (2009).

[39] M. R. Evans and T. Hanney, Nonequilibrium Statistical Mechanics of the Zero-Range Process and Related Models, J. Phys. A 38, R195 (2005).

[40] G. Bianconi and A.-L. Barabási, Bose-Einstein Condensation in Complex Networks, Phys. Rev. Lett. 86, 5632 (2001).

[41] D. Vorberg, W. Wustmann, R. Ketzmerick, and A. Eckardt, Generalized Bose-Einstein Condensation into Multiple States in Driven-Dissipative Systems, Phys. Rev. Lett. 111, 240405 (2013).

[42] D. Vorberg, W. Wustmann, H. Schomerus, R. Ketzmerick, and A. Eckardt, Nonequilibrium Steady States of Ideal Bosonic and Fermionic Quantum Gases, Phys. Rev. E 92, 062119 (2015).

[43] H. Haken and H. Sauermann, Nonlinear Interaction of Laser Modes, Z. Phys. 173, 261 (1963).

[44] M. M Tehrani and L. Mandel, Coherence Theory of the Ring Laser, Phys. Rev. A 17, 677 (1978).

[45] M. Khanbekyan, H. A. M. Leymann, C. Hopfmann, A. Foerster, C. Schneider, S. Höfling, M. Kamp, J. Wiersig, and S. Reitzenstein, Unconventional Collective NormalMode Coupling in Quantum-Dot-Based Bimodal Microlasers, Phys. Rev. A 91, 043840 (2015).

[46] C. Redlich, B. Lingnau, S. Holzinger, E. Schlottmann, S. Kreinberg, C. Schneider, M. Kamp, S. Höfling, J. Wolters, S. Reitzenstein, and K. Lüdge, Mode-Switching Induced Super-Thermal Bunching in Quantum-Dot Microlasers, New J. Phys. 18, 063011 (2016).

[47] M. Fanaei, A. Foerster, H. A. M. Leymann, and J. Wiersig, Effect of Direct Dissipative Coupling of Two Competing Modes on Intensity Fluctuations in a Quantum-DotMicrocavity Laser, Phys. Rev. A 94, 043814 (2016). 
[48] D. N. Krizhanovskii, S. S. Gavrilov, A. P. D. Love, D. Sanvitto, N. A. Gippius, S. G. Tikhodeev, V. D. Kulakovskii, D. M. Whittaker, M. S. Skolnick, and J.S. Roberts, SelfOrganization of Multiple Polariton-Polariton Scattering in Semiconductor Microcavities, Phys. Rev. B 77, 115336 (2008).

[49] G. Dagvadorj, J. M. Fellows, and S. Matyjaśkiewicz, F. M. Marchetti, I. Carusotto, and M. H. Szymańska, Nonequilibrium Phase Transition in a Two-Dimensional Driven Open Quantum System, Phys. Rev. X 5, 041028 (2015).

[50] E. Altman, L. M. Sieberer, L. Chen, S. Diehl, and J. Toner, Two-Dimensional Superfluidity of Exciton Polaritons Requires Strong Anisotropy, Phys. Rev. X 5, 011017 (2015).

[51] P. Lett, Investigation of First-Passage-Time Problems in the Two-Mode Dye Laser, Phys. Rev. A 34, 2044 (1986).

[52] R. Roy, R. Short, J. Durnin, and L. Mandel, First-PassageTime Distributions under the Influence of Quantum Fluctuations in a Laser, Phys. Rev. Lett. 45, 1486 (1980).

[53] K. P. N. Murthy and S. Dattagupta, Monte Carlo Calculations of Switching-Time Statistics in a Two-Mode Laser, Phys. Rev. A 32, 3481 (1985).

[54] C. Kistner, T. Heindel, C. Schneider, A. Rahimi-Iman, S. Reitzenstein, S. Höfling, and A. Forchel, Demonstration of Strong Coupling via Electro-optical Tuning in High-Quality QD-Micropillar Systems, Opt. Express 16, 15006 (2008).

[55] C. Böckler, S. Reitzenstein, C. Kistner, R. Debusmann, A. Löffler, T. Kida, S. Höfling, A. Forchel, L. Grenouillet, J. Claudon et al., Electrically Driven High-Q Quantum DotMicropillar Cavities, Appl. Phys. Lett. 92, 091107 (2008).

[56] S. Reitzenstein, C. Hofmann, A. Gorbunov, M. Strau, S. H. Kwon, C. Schneider, A. Löffler, S. Höfling, M. Kamp, and A. Forchel, AlAsGaAs Micropillar Cavities with Quality Factors Exceeding 150.000, Appl. Phys. Lett. 90, 251109 (2007).

[57] S. M. Ulrich, C. Gies, S. Ates, J. Wiersig, S. Reitzenstein, C. Hofmann, A. Löffler, A. Forchel, F. Jahnke, and P. Michler, Photon Statistics of Semiconductor Microcavity Lasers, Phys. Rev. Lett. 98, 043906 (2007).

[58] J. Wiersig, S. W. Kim, and M. Hentschel, Asymmetric Scattering and Nonorthogonal Mode Patterns in Optical Microspirals, Phys. Rev. A 78, 053809 (2008).

[59] J. Wiersig, Structure of Whispering-Gallery Modes in Optical Microdisks Perturbed by Nanoparticles, Phys. Rev. A 84, 063828 (2011).
[60] B. Peng, Ş. K. Özdemir, M. Liertzer, W. Chen, J. Kramer, H. Yllmaz, J. Wiersig, S. Rotter, and L. Yang, Chiral Modes and Directional Lasing at Exceptional Points, Proc. Natl. Acad. Sci. U.S.A. 113, 6845 (2016).

[61] A. E. Siegman, The Laser Threshold Region, in Lasers (University Science Books, Sausalito, CA, 1986), pp. 516-519.

[62] R. J. Glauber, The Quantum Theory of Optical Coherence, Phys. Rev. 130, 2529 (1963).

[63] S. Strauf, K. Hennessy, M. T. Rakher, Y.-S. Choi, A. Badolato, L. C. Andreani, E. L. Hu, P. M. Petroff, and D. Bouwmeester, Self-Tuned Quantum Dot Gain in Photonic Crystal Lasers, Phys. Rev. Lett. 96, 127404 (2006).

[64] J. Wiersig, C. Gies, F. Jahnke, M. Aßmann, T. Berstermann, M. Bayer, C. Kistner, S. Reitzenstein, C. Schneider, S. Höfling, A. Forchel, C. Kruse, J. Kalden, and D. Hommel, Direct Observation of Correlations Between Individual Photon Emission Events of a Microcavity Laser, Nature (London) 460, 245 (2009).

[65] M. Aßmann, F. Veit, M. Bayer, M. van der Poel, and J. M. Hvam, Higher-Order Photon Bunching in a Semiconductor Microcavity, Science 325, 297 (2009).

[66] H. A. M. Leymann, A. Foerster, and J. Wiersig, Expectation Value Based Equation-of-Motion Approach for Open Quantum Systems: A General Formalism, Phys. Rev. B 89, 085308 (2014).

[67] W. W. Chow, F. Jahnke, and C. Gies, Emission Properties of Nanolasers During the Transition to Lasing, Light Sci. Appl. 3, e201 (2014).

[68] R. Jin, D. Boggavarapu, M. Sargent, P. Meystre, H. M. Gibbs, and G. Khitrova, Photon-Number Correlations Near the Threshold of Microcavity Lasers in the Weak-Coupling Regime, Phys. Rev. A 49, 4038 (1994).

[69] This double-peak structure can be associated with mode switching in the time domain [46,51].

[70] A. Jechow, M. Seefeldt, H. Kurzke, A. Heuer, and R. Menzel, Enhanced Two-Photon Excited Fluorescence from Imaging Agents Using True Thermal Light, Nat. Photonics 7, 973 (2013).

[71] X. M. Lv, Y. D. Yang, L. X. Zou, H. Long, J. L. Xiao, Y. Du, and Y.Z. Huang, Mode Characteristics and Optical Bistability for AlGaInAs/InP Microring Lasers, IEEE Photonics Technol. Lett. 26, 1703 (2014). 